OPEN ACCESS

Edited by:

Christian Gonzalez-Billault, Universidad de Chile, Chile

Reviewed by:

Jaerak Chang,

Ajou University, South Korea

Ann C. Morris

University of Kentucky, United States

${ }^{*}$ Correspondence:

Tao P. Zhong

taozhong@fudan.edu.cn; tzhong@bio.ecnu.edu.cn

Daqing Jin

dqjin@fudan.edu.cn,

dqjin@bio.ecnu.edu.cn

Received: 24 December 2018

Accepted: 29 April 2019

Published: 24 May 2019

Citation:

Li G, Jin D and Zhong TP (2019)

Tubgcp3 Is Required for Retinal Progenitor Cell Proliferation During

Zebrafish Development.

Front. Mol. Neurosci. 12:126.

doi: 10.3389/fnmol.2019.00126

\section{Tubgcp3 Is Required for Retinal Progenitor Cell Proliferation During Zebrafish Development}

\author{
Guobao Li, ${ }^{1,2}$, Daqing Jin ${ }^{1,2 *}$ and Tao P. Zhong ${ }^{1,2 *}$ \\ ${ }^{1}$ State Key Laboratory of Genetic Engineering, School of Life Sciences, Zhongshan Hospital, Fudan University, Shanghai, \\ China, ${ }^{2}$ Shanghai Key Laboratory of Regulatory Biology, Institute of Molecular Medicine, School of Life Sciences, East China \\ Normal University, Shanghai, China
}

The centrosomal protein $\gamma$-tubulin complex protein 3 (Tubgcp3/GCP3) is required for the assembly of $\gamma$-tubulin small complexes ( $\gamma$-TuSCs) and $\gamma$-tubulin ring complexes ( $\gamma$-TuRCs), which play critical roles in mitotic spindle formation during mitosis. However, its function in vertebrate embryonic development is unknown. Here, we generated the zebrafish tubgcp3 mutants using the CRISPR/Cas9 system and found that the tubgcp3 mutants exhibited the small eye phenotype. Tubgcp3 is required for the cell cycle progression of retinal progenitor cells (RPCs), and its depletion caused cell cycle arrest in the mitotic (M) phase. The M-phase arrested RPCs exhibited aberrant monopolar spindles and abnormal distributed centrioles and $\gamma$-tubulin. Moreover, these RPCs underwent apoptosis finally. Our study provides the in vivo model for the functional study of Tubgcp3 and sheds light on the roles of centrosomal $\gamma$-tubulin complexes in vertebrate development.

Keywords: $\gamma$-TuSC, $\gamma$-TuRC, tubgcp3, cell cycle, ciliary marginal zone, zebrafish

\section{INTRODUCTION}

Centrosome, the major microtubule-organizing center (MTOC) in vertebrate cells, provides a major site for microtubule (MT) nucleation and plays key roles in bipolar spindle assembly during mitosis (Kellogg et al., 1994). A typical centrosome consists of a pair of centrioles surrounded by the pericentriolar matrix (PCM) (Bornens, 2002). Many proteins, including $\gamma$-tubulin (GCP1), $\gamma$-tubulin complex proteins (GCPs) and a large number of other centrosome-associated proteins, localize to PCM and are involved in the formation of mitotic spindles. Together with other GCPs, $\gamma$-tubulin forms two distinctly sized complexes: the $\gamma$-tubulin small complex $(\gamma$-TuSC) and the $\gamma$-tubulin ring complex $(\gamma$-TuRC). The $\gamma$-TuSC is a heterotetramer consisting of two copies of $\gamma$-tubulin and one copy each of GCP2 and GCP3/Tubgcp3. Seven $\gamma$-TuSCs with GCP4, GCP5, GCP6 and other accessory proteins assemble into the $\gamma$-TuRC, which facilitates MT nucleation by capping the minus ends of MTs and protecting them from depolymerization (Zheng et al., 1995). Centrosomes have critical roles in brain development, and mutations in genes encoding for centrosome-associated proteins have been shown to be genetically linked to neurodevelopmental disorders (Novorol et al., 2013; Chavali et al., 2014; Morris-Rosendahl and Kaindl, 2015; Buchwalter et al., 2016).

Many studies have shown that $\gamma$-TuSC is involved in MT nucleation and mitotic spindle assembly, and every component of $\gamma$-TuSC is indispensable for cell cycle progression 
(Oakley et al., 1990; Geissler et al., 1996; Knop et al., 1997; Yuba-Kubo et al., 2005; Xiong and Oakley, 2009; Pouchucq et al., 2018). In Saccharomyces cerevisiae, cells overexpressing the wild-type Spc98p/GCP3 or carrying the temperature-sensitive allele spc98-1 arrest in mitosis with a defective spindle (Geissler et al., 1996). The duplication and separation of spindle pole body (SPB; yeast centrosome) are not affected in these cells. Disruption of gcpC/GCP3 in Aspergillus nidulans results in absent functional spindles and defective mitosis (Xiong and Oakley, 2009). In Drosophila mutants for disks-degenerate 4 (dd4, which encodes GCP3), cells exhibit reduced density of spindle microtubules and delayed cell cycle progression from mitosis (Barbosa et al., 2000). Centriole duplication and separation are defective in some of the $d d 4$ mutant cells. In addition, $\gamma$-tubulin is missing from the spindle poles and becomes dispersed throughout the cell. Mitotic arrest has been observed in Hela, T98, and U87MG cell lines after depletion of GCP3 using siRNA (Draberova et al., 2015; Farache et al., 2016; Cota et al., 2017). However, Mikule et al. (2007) found that cells harboring wild-type p53 (RPE-1, BJ-1, HME-1, and HCT-116) arrest in G1 phase in a p53-dependent manner after being transfected with siRNA against GCP3. Given that U87MG cells expressing wild-type p53 show mitotic arrest, it remains unclear which types of cell cycle defects will happen in the GCP3-deleted cells with wild-type p53 gene. These different cell cycle defects may occur in a cell type-specific manner as these studies were carried out using different cell lines. Moreover, there have been no functional studies of GCP3 in vivo in a vertebrate system.

Among vertebrate models, the zebrafish provides many unique advantages over other rodents for gene functional study during early embryonic development. For example, owing to its external fertilization and rapid development, zebrafish early embryos can be easily visualized and manipulated. In addition, maternal gene products, synthesized during oogenesis and supplied to the egg, play essential roles in the earliest stages of zebrafish embryonic development (Pelegri, 2003). With disruption of any of the maternal-effect genes, the embryos continue to develop until the maternal supply is exhausted, which facilitates the functional study of these genes at relatively late developmental stages. The zebrafish retina is part of the central nervous system (CNS), and its neuroanatomy is well characterized. The ciliary marginal zone (CMZ), a proliferative region located at the periphery of the retina, provides an excellent model for the study of neurodevelopment (Novorol et al., 2013). The retina grows continuously throughout life and almost all retina neurogenesis comes from $\mathrm{CMZ}$ after the embryogenesis of retina is completed at $60 \mathrm{hpf}$ (Marcus et al., 1999). The CMZ contains retinal stem cells (RSCs) and retinal progenitor cells (RPCs), exhibiting a peripheral-tocentral arrangement pattern. The RSCs were located nearest to the periphery, the proliferative RPCs resided in the middle, and the post-mitotic RPC cells were positioned at the most central of the CMZ (Wehman et al., 2005; Cerveny et al., 2010; Valdivia et al., 2016; Wan et al., 2016). In CMZ, cell proliferation and differentiation are precisely coordinated for the growth of zebrafish eyes. Many cell proliferation and differentiation-associated genes have been studied using the zebrafish model (Wehman et al., 2005; Cerveny et al., 2010; Valdivia et al., 2016).

In this study, we explored the in vivo function of tubgcp3 using the zebrafish model. We found that Tubgcp3 is essential for the development of zebrafish retina. Knockout of the tubgcp3 gene resulted in the small eye phenotype exhibiting CMZ defects due to the abnormal cell cycle progression. Depletion of Tubgcp3 in RPCs caused mitotic arrest and apoptosis. Our findings reveal the critical roles of GCP3 in cell cycle progression, providing insights into the function of its associated complexes, $\gamma$-TuSC and $\gamma$-TuRC, in development, and establish a vertebrate model for further study.

\section{MATERIALS AND METHODS}

\section{Zebrafish Maintenance}

Zebrafish (Danio rerio) were maintained and bred under standard conditions as previously described (Westerfield, 2000). Stages of embryonic development were determined according to their morphology (Westerfield, 2000). Embryos were treated with $0.003 \%$ 1-phenyl-2-thiourea (PTU) in egg water to prevent the production of pigment. The transgenic line $\mathrm{Tg}(\mathrm{HuC}$ :GFP) was used in this study, in which the zebrafish brain is labeled with green fluorescent protein (GFP) (Park et al., 2000). All animal experiments were approved by the Institutional Animal Care and Use Committee, Fudan University.

\section{Generation of tubgcp3 Mutants by the CRISPR/Cas9 System and Phenotypic Analysis}

Disruption of tubgcp3 transcript variant X1 (GenBank accession number XM_005170995) was performed using CRISPR/Cas9 technology. Cas9 nuclease target sites were designed using the ZiFiT Targeter online software. The target site in the exon 4 was selected and the corresponding sequence was 5'-GGTCCTCACAGAGGCTGAGCAGG-3'. Cas9 mRNA and guide RNA (gRNA) were synthesized using the mMESSAGE mMACHINE T7 Transcription Kit (AM1344, Invitrogen, United States) and MAXIscript T7 Transcription Kit (AM1322, Invitrogen, United States), respectively. Then, 300 pg Cas 9 mRNA and 30 pg gRNA were co-injected into the one-cell stage zebrafish embryos to knockout the tubgcp3 gene. The PCR primers for genotyping were as follows: forward (5'-ATTACGCAGAGGACCAAGA-3') and reverse (5'-AAAATAGGATTTCATACAGGAACCCG-3'). The tubgcp3 mutants were identified at $3 \mathrm{dpf}$ by the developmental defects of the head and the arch. Heterozygous animals were incrossed to generate embryos for experimental analyses. Homozygous mutants were used as the experimental group and siblings as the control in this study.

For morphological measurements, embryos were anesthetized with $0.16 \mathrm{mg} / \mathrm{mL}$ Tricaine (A5040, Sigma-Aldrich) and embedded in 3\% methylcellulose (M0387, Sigma-Aldrich). Body 
length was measured laterally in the rostral-caudal axis. Eye size was measured laterally along the longest diameter of the eye. Brain size was measured by calculating the dorsal area of the brain using the transgenic line $\mathrm{Tg}(\mathrm{HuC}$ :GFP) (Park et al., 2000).

For cartilage staining, $5 \mathrm{dpf}$ embryos were fixed in Bouin's solution overnight. The fixed embryos were washed three times in $70 \%$ ethanol/0.1\% ammonia, then washed two more times in $95 \%$ ethanol. The embryos were transferred into Alcian blue solution ( $80 \%$ ethanol $/ 20 \%$ glacial acetic acid, $0.1 \%$ Alcian blue) and stained overnight. After staining, the embryos were gradually rehydrated in a decreasing ethanol series $(60 \%, 40 \%$, and $20 \%$ ) and washed twice in phosphate-buffered saline (PBS). The embryos were incubated in $3 \%$ hydrogen peroxide/1\% potassium hydroxide until the pigmentation was removed. After that, the samples were incubated in $0.05 \%$ trypsin in $30 \%$ sodium tetraborate until cleared. Then, the embryos were transferred into $70 \%$ glycerol for imaging.

\section{Semi-Quantitative RT-PCR and Plasmids}

Total RNA was extracted from whole embryos at the indicated developmental stages using a TRIZOL reagent. cDNA synthesis was carried out using a TOYOBO RT-PCR kit (FSQ-301, TOYOBO, Japan) according to the manufacturer's instructions. The expression profile of tubgcp 3 was analyzed using the following primers: tubgcp3 forward (5'-AGAAGAGATGGCCGAGTGGG-3') and reverse $\left(5^{\prime}\right.$-CGCCAGACGAGTTCTGAGTA- $\left.3^{\prime}\right) ; \quad \beta$-actin forward (5'-CAGCCTTCCTTCCTGGGTAT-3') and reverse $\left(5^{\prime}\right.$-GCCATACAGAGCAGAAGCCA- $\left.3^{\prime}\right)$. cDNA encoding full-length $\gamma$-tubulin was amplified by PCR and inserted into pEGFP-N2 (Clontech, United States) using EcoR I and Xho I. cDNA encoding Full-length, N-terminal (Tubgcp3 1-551 aa) and C-terminal (Tubgcp3 552-906 aa) Tubgcp3 were amplified by PCR and cloned into pCMV-Myc (Clontech, United States) using EcoRI and BamHI.

\section{In situ Hybridization Analyses}

In situ hybridization was performed on $10 \mu \mathrm{m}$ cryosections of zebrafish eyes. Briefly, zebrafish embryos were fixed in $4 \%$ paraformaldehyde overnight at $4^{\circ} \mathrm{C}$. The next day, the fixed embryos were washed three times with PBS and dehydrated in $30 \%$ sucrose. Then, the embryos were embedded in OCT (Invitrogen) before being sectioned at $10 \mu \mathrm{m}$ with a Leica cryostat (Leica CM1860, Leica Microsystems, Germany). For whole-mount in situ hybridization, the steps were performed as previously described (Thisse and Thisse, 2008).

To generate in situ probes, PCR products of $\gamma$-tubulin, tubgcp2, tubgcp3, pcna, vsx2, col15a1b, ccnd1, atoh7, and $c d k n 1 c$ were amplified from cDNA and subcloned into pGEM-T Easy vector (A1360, Promega). Probe plasmids were digested with restriction enzyme to make DNA templates. Digoxigenin-labeled RNA probes were generated by in vitro transcription using the MAXIscript SP6/T7 Transcription Kit (AM1322, Invitrogen). In situ hybridization signals were detected by using NBT/BCIP (11681451001, Roche) for sections and BM-Purple (11442074001, Roche) for whole-mount embryos.

\section{BrdU Incorporation}

To examine RPC proliferation, $66 \mathrm{hpf}$ embryos were incubated in the thymidine analog 5-bromo-2-deoxyuridine (BrdU) (B5002, Sigma-Aldrich) at $10 \mathrm{mM} / 1 \%$ dimethyl sulfoxide (276855, Sigma-Aldrich) in embryo medium for $6 \mathrm{~h}$ at $28.5^{\circ} \mathrm{C}$. Then, the embryos were rinsed twice with PBS before being fixed at $72 \mathrm{hpf}$. To examine RSC proliferation, $4 \mathrm{dpf}$ embryos were incubated with $3 \mathrm{mM}$ BrdU/1\% dimethyl sulfoxide in embryo medium for $24 \mathrm{~h}$ before being fixed at $5 \mathrm{dpf}$.

\section{HE Staining}

For Hematoxylin and Eosin (HE) staining, embryos were dehydrated using a graded ethanol series $(50,70,90,95$, and $100 \%$ ) before being transferred to xylene. Then, the embryos were embedded in paraffin wax and sectioned at $5 \mu \mathrm{m}$ using a Leica Cryostat (Leica RM2235, Leica). HE staining was performed according to standard protocols.

\section{Immunofluorescence and TUNEL Assay}

Embryos were fixed in $4 \%$ paraformaldehyde for $2 \mathrm{~h}$ at room temperature (RT) except for anti- $\gamma$-tubulin staining fixed in $4 \%$ formalin. Cryosections were prepared as described in the previous ISH section. Cryosections were boiled in $0.01 \mathrm{M}$ citric acid ( $\mathrm{pH} \mathrm{6)}$ ) for $30 \mathrm{~min}$. After being washed three times in PBST $(0.1 \%$ Triton $\mathrm{X}-100$ and $0.1 \%$ Tween 20 in PBS), the sections were incubated in blocking solution (2\% horse serum, 10\% FBS, $0.1 \%$ Triton X-100, $0.1 \%$ Tween 20, 10\% DMSO in PBS) for $60 \mathrm{~min}$. The sections were then incubated with primary antibodies at $4^{\circ} \mathrm{C}$ overnight. Primary antibodies used in this study include rabbit anti-phosphohistone-H3 (Ser-10) (06-570, Millipore; 1:400), mouse anti-BrdU (11170376001, Roche; 1:300), rabbit anti- $\gamma \mathrm{H} 2 \mathrm{AX}$ (Ser-139) antibody (GTX127342, GeneTex; 1:300), mouse anti-PCNA (P8825, Sigma-Aldrich; 1:200), mouse anti- $\gamma$-Tubulin antibody (T5326, Sigma-Aldrich; 1:1000), mouse anti- $\alpha$-Tubulin antibody (T9026, Sigma-Aldrich; 1:1000), mouse monoclonal anti-acetylated tubulin (T6793, Sigma-Aldrich; 1:1000), rabbit polyclonal anti-opsin (AB5404, Millipore; 1:1000), mouse monoclonal ZPR-1 (ab174435, Abcam; 1:500) and mouse monoclonal anti-phodopsin 4D2 (ab183359, Abcam; 1:1000). The sections were washed three times in PBST and incubated with secondary antibodies at RT for $2 \mathrm{~h}$. Secondary antibodies were goat anti-mouse Alexa-Fluor-488 (A-11001, Invitrogen; 1:1000), goat anti-mouse Alexa-Fluor-594 (A-21135, Invitrogen; 1:1000), goat anti-mouse Alexa-Fluor-555 (A-21422, Invitrogen; 1:1000), goat anti-rabbit Alexa-Fluor-488 (A-11034, Invitrogen; 1:1000) and goat anti-rabbit Alexa-Fluor-594 (A-11037, Invitrogen; 1:1000). Sections were counterstained with 4',6-diamidino-2-phenylindole (DAPI) (D3571, Invitrogen) when needed. The filamentous actin (F-actin) was stained with Alexa Fluor 488 phalloidin (A12379, Thermo Fisher; 1:100). For BrdU staining, sections were treated with $2 \mathrm{M}$ $\mathrm{HCl}$ for $1 \mathrm{~h}$ at $\mathrm{RT}$ before being incubated in blocking solution. For whole-mount immunostaining, the $4 \%$ paraformaldehyde fixed embryos were permeabilized 
by proteinase $\mathrm{K}$ digestion before being incubated in blocking solution.

To detect cell death, a TUNEL assay was performed on sections using the In Situ Cell Death Detection Kit (Roche) according to the manufactures' instruction.

\section{Immunoprecipitation and Western Blotting}

For immunoprecipitation, HEK293T cells were co-transfected with GFP-tagged zebrafish $\gamma$-tubulin and Myc-tagged zebrafish Tubgcp3 constructs using Lipofectamine 2000 Transfection Reagent (Invitrogen). After $48 \mathrm{~h}$, transfected cells were lysed with lysis buffer (50 mM HEPES, pH 7.5, $150 \mathrm{mM} \mathrm{NaCl}, 1 \mathrm{mM}$ $\mathrm{MgCl}_{2}, 1 \mathrm{mM}$ EGTA, $0.5 \%$ NP-40, protease inhibitor cocktail (04693132001, Roche)) and incubated on ice for $10 \mathrm{~min}$. The lysate was obtained by centrifugation at $16,000 \times g$ for $15 \mathrm{~min}$ at $4^{\circ} \mathrm{C}$. The supernatants were incubated with anti-Myc antibody and protein A-Sepharose (GE Healthcare) at $4^{\circ} \mathrm{C}$ for $4 \mathrm{~h}$. Then the precipitants were washed three times with lysis buffer before being boiled in SDS loading buffer for immunoblot. Zebrafish embryos (5 dpf) were collected, washed and homogenized in RIPA lysis buffer (P0013B, Beyotime Biotechnology, China) containing protease inhibitor cocktail (04693132001, Roche). The following primary antibodies were used: rabbit anti-Myc (A5598, Sigma-Aldrich; 1:1000), rabbit anti-GFP (50430-2-AP, ProteinTech; 1:2000), rabbit anti-TUBGCP3 (15719-1-AP, ProteinTech; 1:1000), mouse anti- $\beta$-Actin (CW0264M, CWBIO; $1: 2000)$ and rabbit anti- $\beta$-Actin (GTX124388, GeneTex; 1:5000).

\section{Senescence-Associated $\beta$-Galactosidase (SA- $\beta$-gal) Assay}

To detect cellular senescence, $\beta$-galactosidase assay was performed using the Senescence Cells Histochemical Staining Kit (CS0030, Sigma-Aldrich). Zebrafish embryos (5 dpf) were fixed in $0.2 \%$ glutaraldehyde at RT for $2 \mathrm{~h}$. The fixed embryos were washed three times in PBS before being embedded in OCT (Invitrogen). Then, the embryos were sectioned at $10 \mu \mathrm{m}$ for the assay.

\section{Imaging}

The images of in situ hybridization, HE staining and SA- $\beta$-gal assay were captured using a Nikon ECLIPSE Ni microscope (Nikon) or Olympus microscope (IX83, Olympus). Immunostaining images were acquired using a Zeiss Axio Observer.Z1 microscope (Zeiss) or Nikon A1 confocal microscope (Nikon). Bright-field images of whole mount zebrafish embryos were taken using a Leica microscope (Leica M205FA, Leica).

\section{Statistical Analyses}

To calculate cell proliferation and cell death in zebrafish retina, sections of the most central portion of the retina were selected for the analysis. The cell number was manually counted using Image J software within a defined region in the nasal part of the retina including the nasal CMZ $(140 \mu \mathrm{m} \times 100 \mu \mathrm{m})$. Proliferating cells in embryo tails was counted within a defined region including $\sim 400 \mu \mathrm{m}$ of the tails. Statistical analyses were performed with unpaired Student's $t$-test using GraphPad Prism software. A $P$-value $<0.05$ was considered statistically significant $\left({ }^{*} P<0.05,{ }^{* *} P<0.01\right)$. Data are expressed as mean + SEM.

\section{RESULTS}

\section{The Expression Pattern of Zebrafish tubgcp3 During Early Development}

To analyze the roles of tubgcp3 during zebrafish early development, we first examined the expression pattern of tubgcp3 by RT-PCR (Figure 1A) and whole-mount in situ hybridization (WISH) (Figures 1B-J). The RT-PCR analyses showed tubgcp3 transcripts were maternally deposited and continually expressed from 2-cell stage to 5 days post fertilization (dpf) during early embryonic development (Figure 1A). The expression of tubgcp3 mRNA first decreased and then increased from 2-cell stage to $24 \mathrm{hpf}$, indicating the degradation of maternally deposited tubgcp 3 transcripts and the activation of zygotic transcripts during the maternal-to-zygotic transition (MZT). Consistent with RT-PCR results, WISH analyses showed that tubgcp 3 mRNA was detected at all developmental stages from cleavage stage to $3 \mathrm{dpf}$ compared with the control group (Figures 1B-J,N, $\mathbf{O}$ ). Furthermore, we found that the expression of tubgcp 3 was enriched in the head and eye as well as weak expression in other tissues of the embryos from 1 to $3 \mathrm{dpf}$ (Figures 1B-J). To further characterize its expression in zebrafish retina, in situ hybridization (ISH) was performed on cryosections of the embryo eyes. In comparison with the sense probe as the control (Figure 1P), tubgcp3 was expressed throughout the retina with a gradual enrichment at CMZ (Figures $1 \mathbf{K}-\mathbf{M}$ ). We also detected the expression of tubgcp 2 and $\gamma$-tubulin, which encodes another two subunits of $\gamma \mathrm{TuSC}$. Their expression was similar to that of tubgcp3 (Supplementary Figures S1A-H). In addition, the expression of pcna, a proliferation marker, was confined in the CMZ from 3 to $5 \mathrm{dpf}$ (Supplementary Figures S1I-L). These data indicate that $t u b g c p 3$ may have a role in cell proliferation during zebrafish retinal development.

\section{Generation of Zebrafish tubgcp3 Mutants and Morphological Analysis}

To investigate the roles of tubgcp3 during zebrafish embryonic development, we established tubgcp 3 knockout zebrafish lines using the CRISPR/Cas9 system. The CRISPR/Cas9 target site was designed in the exon 4 of the tubgcp 3 gene. Finally, two mutant alleles with 5-base pair (bp) deletion and 11-bp insertion were identified (Figure $\mathbf{2 A}$ and data not shown). Since the two mutant lines exhibited the same phenotypes, we used the 5-bp deletion line for further research. The 5-bp deletion was predicted to lead to a premature termination in Tubgcp3 protein translation and yield a 99-amino acid (aa)-truncated Tubgcp3 protein absent of the two conversed GRIP domains (Figure 2B). tubgcp 3 transcripts were detected in the tubgcp 3 mutant retina (Supplementary Figure S2A), which suggested that the mutated tubgcp3 mRNA escaped non-sense-mediated mRNA decay 


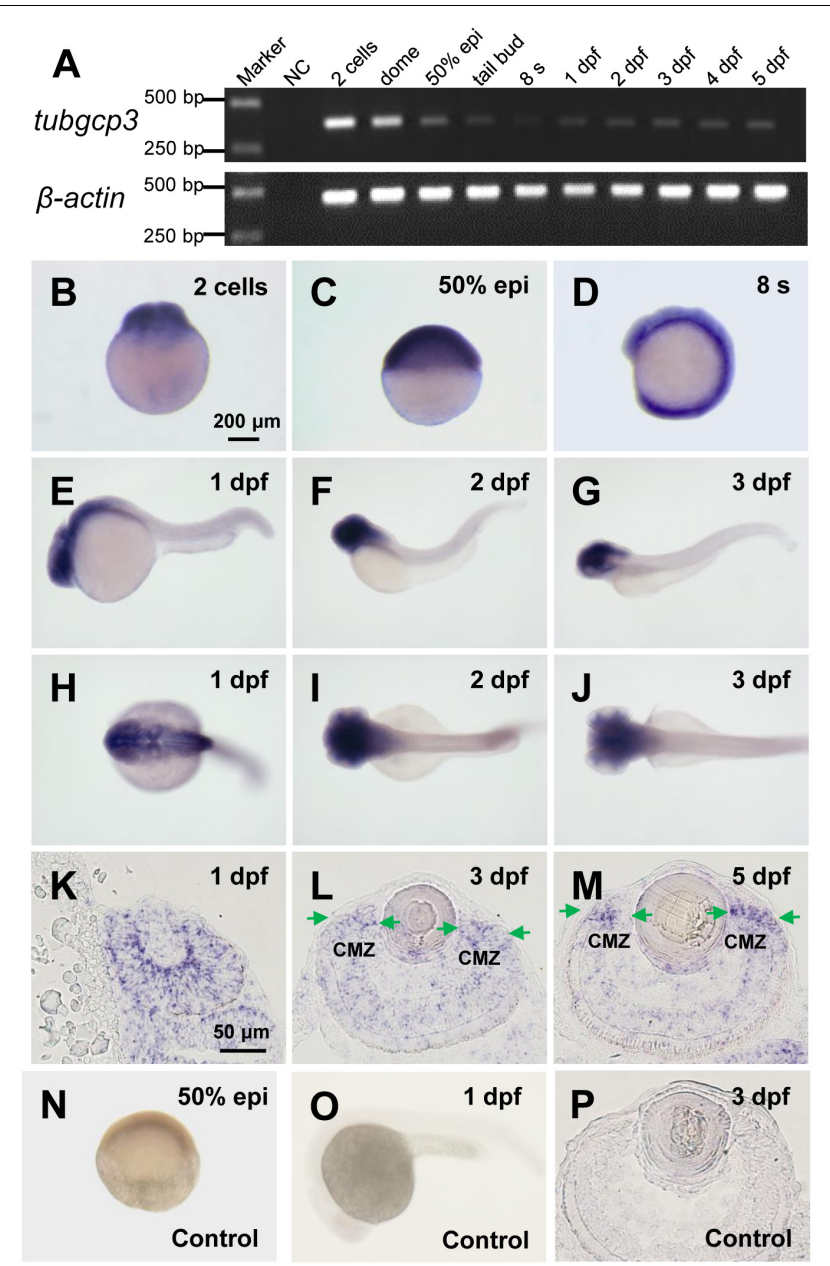

FIGURE 1 | Expression of tubgcp3 during zebrafish early development. (A) Semi-quantitative RT-PCR analyses showing the expression of zebrafish tubgcp3 from 2-cell stage to 5 days post-fertilization (dpf). $\beta$-actin was used as the internal control. (B-D) Whole-mount in situ hybridization (WISH) displaying ubiquitous expression of tubgcp3 at the two-cell stage (B), 50\%-epiboly (C) and 8-somite stage (8 s) (D). (E-J) From 1 to $3 \mathrm{dpf}$, the expression of tubgcp3 becomes concentrated at the head. (K-M) In situ hybridization (ISH) of zebrafish retinal cryosections exhibit the expression of tubgcp3 throughout the whole retina at $1 \mathrm{dpf}(\mathbf{K})$. From $3 \mathrm{dpf}$, its expression is enriched at the ciliary marginal zone (CMZ) (L,M). (N-P) No positive staining is detected with the sense probe. Arrows indicate the $\mathrm{CMZ}$ of the retina. Scale bars: $200 \mu \mathrm{m}$ (B-J,N,O); $50 \mu \mathrm{m}$ (K-M,P).

(NMD). In addition, sequencing analyses confirmed that the 5-bp deletion transcripts existed in tubgcp3 mutants (Supplementary Figure S2B). Furthermore, the full-length Tubgcp3 protein was significantly decreased in mutant embryos (Figure 2C). The residual full-length Tubgcp 3 in tubgcp 3 mutants maybe attributed to the maternal supplied Tubgcp3 that was unusually stable in its associated complexes or depleted at a very low rate in embryos. All these results indicate a loss-of-function mutation in tubgcp3 gene.

The tubgcp 3 mutant embryos were indistinguishable from their wild-type siblings before $2 \mathrm{dpf}$. From 3 to $5 \mathrm{dpf}$, the mutants exhibited a progressed $\mathrm{MCPH}$ (microcephaly)-like phenotype with reduced brain and eye size (Figures 2D,F). We determined the small brain phenotype using transgenic $\mathrm{Tg}(\mathrm{HuC}$ :GFP), in which GFP expression is controlled by the promoter of neuronal gene $\mathrm{HuC}$ (Park et al., 2000). We found that the brain size was reduced about $10 \%$ in the tubgcp 3 mutants compared to the wild-type siblings at $3 \mathrm{dpf}$ (Figure 2G). The brain size reduction in tubgcp 3 mutants became more significant (about $24 \%$ reduction) when measured at $5 \mathrm{dpf}$ (Figure 2G). Notably, the eyes became smaller in the tubgcp3 mutants when compared with wild-type siblings from $3 \mathrm{dpf}$ to $5 \mathrm{dpf}$ (Figures 2E,F). Other defects including dorsal tail curvature (Figure 2D), uninflated swim bladder (Figure 2D), body length reduction (Supplementary Figure S3A) and jaw malformation (Supplementary Figure S3B) were also visible in tubgcp 3 mutants. The developmental defects were gradually more serious, and the mutants died around 10-14 dpf.

\section{The tubgcp3 Mutants Exhibit Abnormal CMZ}

In order to investigate the eye defects in tubgcp 3 mutants in detail, we performed Hematoxylin and Eosin (HE) staining on transverse eye paraffin sections. The tubgcp3 mutant retina showed defective CMZ with disorderly cells when compared to wild-type siblings at $3 \mathrm{dpf}$ (Figures 3A,B,E,F). Notably, these abnormal cells disappeared from the $\mathrm{CMZ}$ at $5 \mathrm{dpf}$ (Figures 3C,D,G,H). However, the laminar structure of central retina appeared normal in tubgcp 3 mutants at $3 \mathrm{dpf}$ and $5 \mathrm{dpf}$ (Figures 3I-L), including the ganglion cell layer (GCL), inner plexiform layer (IPL), inner nuclear layer (INL), outer plexiform layer (OPL), outer nuclear layer (ONL) and retinal pigment epithelium (RPE).

Ciliary marginal zone is responsible for adding new neurons to the continuously growing retina in zebrafish larvae, which consists of retinal stem cells (RSCs) and retinal progenitor cells (RPCs) (Wehman et al., 2005; Cerveny et al., 2010; Valdivia et al., 2016; Wan et al., 2016). vsx2 encodes a homeodomain transcription factor, which is expressed in CMZ cells, Müller glia and a subpopulation of bipolar cells (Vitorino et al., 2009). The expression region of $v s x 2$ was reduced in tubgcp 3 mutant CMZ from 3 to $5 \mathrm{dpf}$ (Figures 4A-D), which was consistent with the development defects detected by $\mathrm{HE}$ staining (Figures $3 \mathrm{E}-\mathbf{H}$ ). col15a1b expressed at the most periphery of $\mathrm{CMZ}$, where it was considered to be a stem-cell niche (the location of RSCs) (Pujic et al., 2006; Cerveny et al., 2010; Gonzalez-Nunez et al., 2010; Valdivia et al., 2016). Its expression seems to be unaffected in the tubgcp3 mutants (Supplementary Figure S4A). ccnd1, a marker for proliferating cells, encodes Cyclin D1 (CCND1) which is required for G1-S transition (Cerveny et al., 2010; Valdivia et al., 2016). The expression of ccnd1 was significantly reduced in tubgcp3 mutant CMZ (Figures 4E-H). atoh7 encodes ATH5, a member of bHLH transcription factor, and is involved in the differentiation of ganglion cells (Masai et al., 2000; Cerveny et al., 2010; Valdivia et al., 2016). $c d k n 1 c$ encodes the p57 cip/kip, a cyclin-dependent kinase inhibitor, which is required for many retinal cells to exit from the cell cycle before their 
A

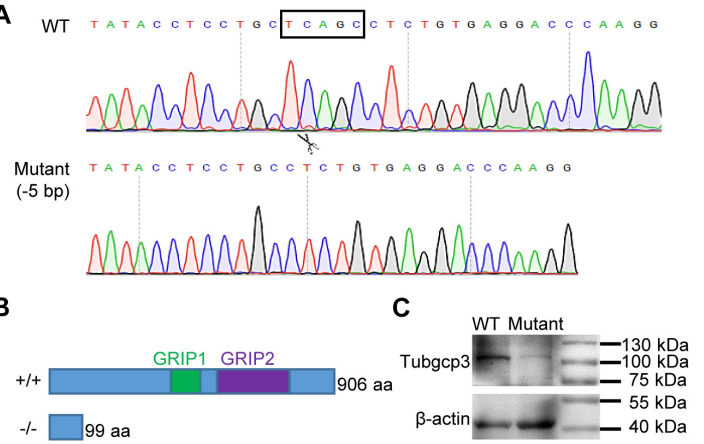

D

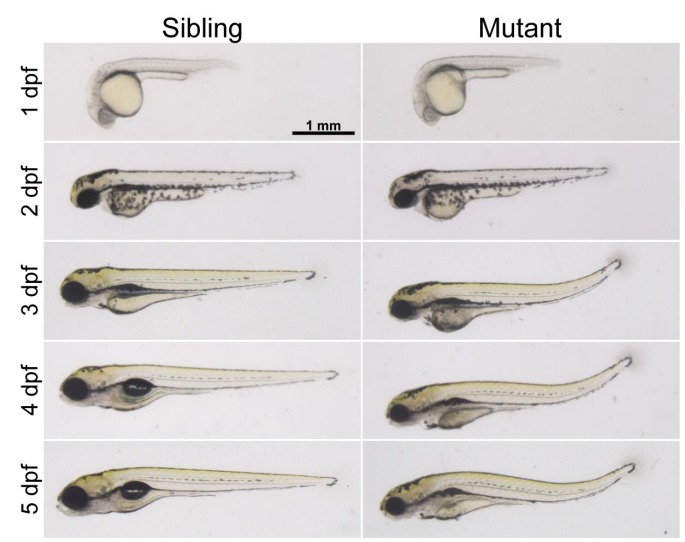

E

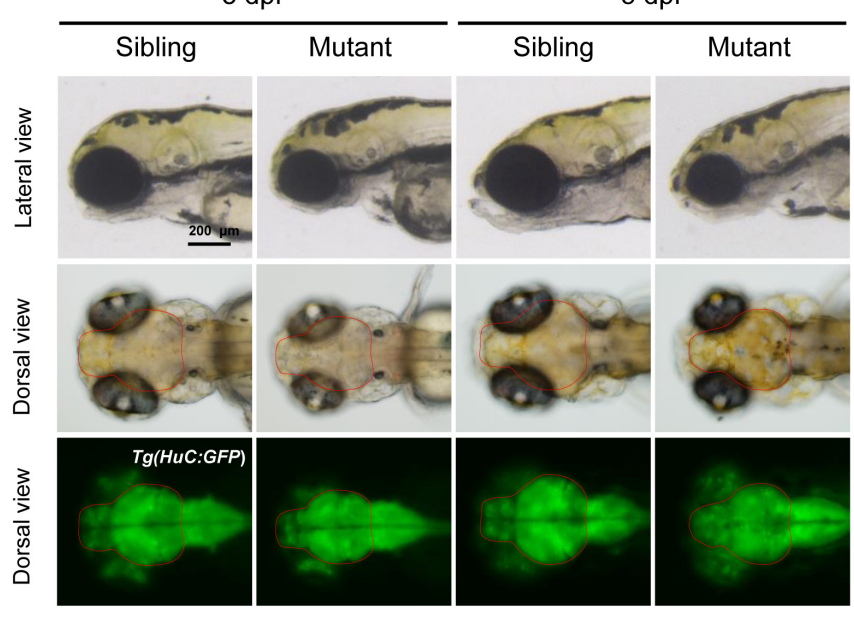

$\mathrm{F}$

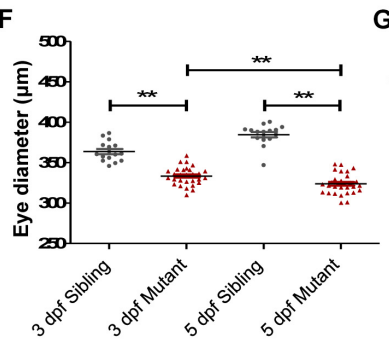

G

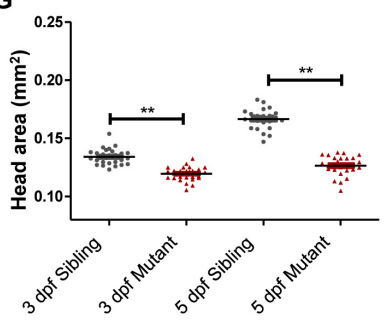

FIGURE 2 | CRISPR/Cas9-mediated tubgcp3 knockout results in zebrafish developmental defects. (A) Sanger sequencing displaying a 5-base pair (bp) deletion in tubgcp3 gene in the zebrafish mutants. The black box indicates the deletion. (B) Predicted structure and amino acid sequence of the wild-type and the mutant alleles of Tubgcp3. The 5-bp deletion in tubgcp3 gene was predicted to generate a 99 amino acid (aa)-truncated Tubgcp3 protein without the GRIP1 domain (green box) and GRIP2 domain (purple box). (C) Western blotting analysis showing Tubgcp3 protein decreased in tubgcp3 mutant embryos at 5 dpf. (D) Whole-mount lateral views of the tubgcp3 mutant and sibling embryos at the indicated developmental stages. (E) Higher magnification of the lateral and dorsal views of zebrafish heads from siblings and tubgcp3 mutants at 3 and $5 \mathrm{dpf}$. The brain size is measured based on the fluorescent area in the head of $T g(H u C: G F P)$. Red dotted lines indicate the brain area of the embryos used for analysis. (F,G) Scatter plot of eye and head size from wild-type siblings and tubgcp3 mutants at 3 and 5 dpf. Data are from 29 embryos for each group. Student's $t$-test: ${ }^{* *} P<0.01$. Scale bars: $1 \mathrm{~mm}$ (D); $200 \mu \mathrm{m}$ (E).

further differentiation (Ohnuma et al., 1999; Shkumatava and Neumann, 2005; Cerveny et al., 2010; Valdivia et al., 2016). The expression of atoh 7 and $c d k n 1 c$ was significantly reduced in tubgcp3 mutant CMZ at $3 \mathrm{dpf}$ and almost disappeared at $5 \mathrm{dpf}$ (Figures 4I-P). These results reveal that the tubgcp3 mutants suffer from a significant reduction in proliferating and differentiating in CMZ cells.

In addition, different retinal cell types, including Müller glia cells (expressing vs $x 2$ ) (Figures 4A-D), a subset of bipolar cells (expressing vs $x 2$ ) (Figures 4A-D), cones (labeled with anti-ZPR1 for double cone photoreceptors and anti-M-opsin for cone outer segments) (Figures 4Q-T and Supplementary Figure S4B) and rods (labeled with anti-4D2 for rod outer segments) (Supplementary Figure S4B), appeared normal in the tubgcp3 mutants. Moreover, cilia in photoreceptor cells (labeled with anti-acetylated $\alpha$-tubulin) and retinal laminar structures, including the inner segment, inter plexiform layer (IPL), the outer limiting membrane (OLM) and the outer plexiform layer (OPL) (labeled with phalloidin) (Supplementary Figure S4B and data not shown), were also normal in tubgcp3 mutants. These results suggest that these differentiated cells and structures are unaffected in the central reitna of the tubgcp3 mutants.

\section{Loss of Tubgcp3 in CMZ Cells Causes M-Phase Arrest}

Given the reduction of cell proliferation and differentiation in the tubgcp3 mutant CMZ (Figures 4E-P), we first examined whether cell cycle progression was defective in mutant retinae using BrdU incorporation as an S phase marker and Phospho-Histone $\mathrm{H} 3$ (PH3) as a mitotic marker. Considering the length of the cell cycle in the CMZ at early larval stages is about 6 to $8 \mathrm{~h}$ (Li et al., 2000; Wehman et al., 2007), zebrafish embryos were incubated with BrdU from 66 to $72 \mathrm{hpf}$ and then subjected to section and cell cycle analysis. Cycling RPCs would be labeled by BrdU except those that had passed $S$ phase and would exit cell cycle and undergo differentiation.

We compared expression patterns of BrdU and $\mathrm{PH} 3$ between the tubgcp 3 mutants and wild-type siblings. In wild-type siblings, the entire CMZ was labeled with BrdU with about three PH3+ cells scattered along the CMZ (Figures 5A,C,E,G). In the tubgcp3 


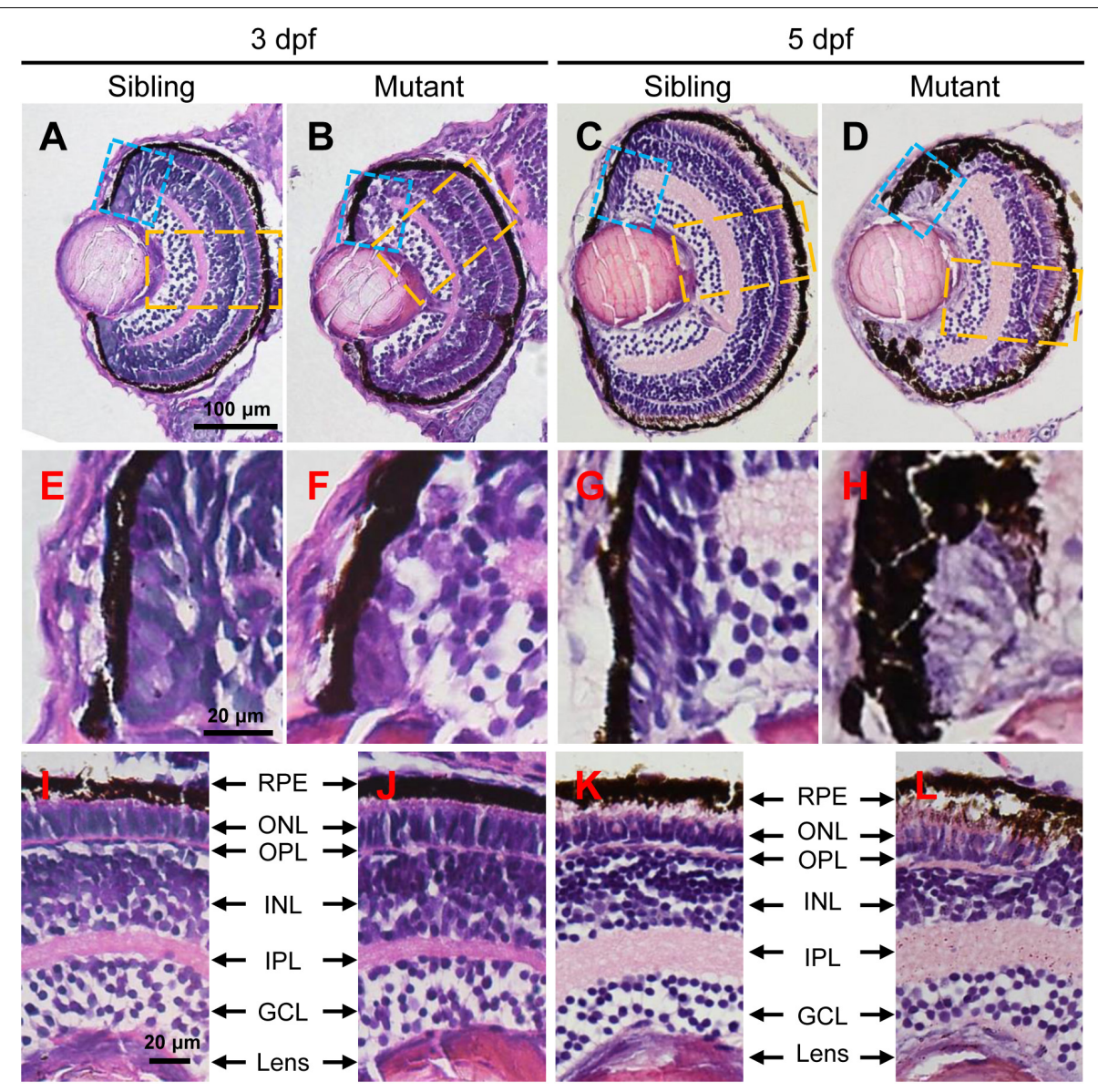

FIGURE 3 | The tubgcp3 mutants exhibit developmental defects in the CMZ of the retina. (A-D) Hematoxylin and Eosin (HE) staining displaying significant defects in the $\mathrm{CMZ}$ of the tubgcp3 mutant retinae at $3 \mathrm{dpf}$ and $5 \mathrm{dpf}$. (E-H) Higher-magnification images of the CMZ in blue dotted rectangles in (A-D). (I-L) Higher-magnification image of the orange dotted rectangles in (A-D). The tubgcp3 mutants exhibit normal retinal laminar structures in the central retina. GCL, ganglion cell layer; IPL, inner plexiform layer; INL, inner nuclear layer; OPL, outer plexiform layer; ONL, outer nuclear layer; RPE, retinal pigment epithelium. Scale bars: $100 \mu \mathrm{m}$ (A-D); $20 \mu \mathrm{m}$ (E-L).

mutants, there was a significantly increased number of $\mathrm{PH} 3+$ cells in CMZ and its adjacent regions (Figures 5B,F,G). We noticed that a portion of these $\mathrm{PH} 3+$ cells were BrdU negative (Figure 5F). In contrast, there were no $\mathrm{PH} 3+\mathrm{BrdU}$ - cells in wild-type siblings (Figure 5E). In addition, BrdU+ cells were significantly reduced in mutant CMZ (Figures 5D,F,G). The $\mathrm{PH} 3+$ BrdU- cells in tubgcp3 mutants had passed S phase when we performed BrdU treatment, and they then went into mitosis as they were stained by $\mathrm{PH} 3$. However, these cells were still $\mathrm{PH} 3+$ after the $6 \mathrm{~h}$ BrdU treatment, which indicates that they were still in the mitotic (M) phase. Since G2 and M phase are shorter than $6 \mathrm{~h}$, the results suggest that these RPCs were arrested in $\mathrm{M}$-phase. Moreover, there were also more BrdU+ $\mathrm{PH} 3+$ cells in the tubgcp3 mutants than in wild-type siblings (Figures 5E,F,G), suggesting that some of the cycling RPCs were arrested in $\mathrm{M}$-phase after they passed the $\mathrm{S}$ phase. When whole-mount immunostaining was carried out under the same BrdU treatment condition, we observed similar results that a large number of $\mathrm{PH} 3+\mathrm{BrdU}$ - cells existed in the tubgcp 3 mutant tails while they absent in wild-type siblings (Supplementary
Figures S5A,B). These results suggest that Tubgcp3 is required for RPCs to go through mitosis.

Given the mitotic arrested cells detected in the tubgcp 3 mutant retinae, we analyzed the spindle formation by immunostaining using anti- $\alpha$-tubulin and anti-PH3 antibodies. In wild-type siblings, bipolar mitotic spindles were formed in mitotic cells (Figures 5J,L). In contrast, abnormal monopolar spindles were observed in the mitotic arrested cells in tubgcp 3 mutant retina (Figures $\mathbf{5 K}, \mathbf{M}, \mathbf{N}$ ). The microtubules arrayed radially in the center and the condensed chromosomes located at the periphery in these cells (Figures $\mathbf{5} \mathbf{I}, \mathbf{K}, \mathbf{M}$ ). These results suggest that depletion of Tubgcp 3 causes a defect in mitotic spindle formation in RPCs, resulting in mitotic arrest in these cells.

\section{Tubgcp3 Deficiency Impairs Centrioles Distribution}

Since centriole duplication and segregation are essential for bipolar spindle formation during mitosis, we examined whether these processes were affected in the tubgcp 3 mutant retinal cells 


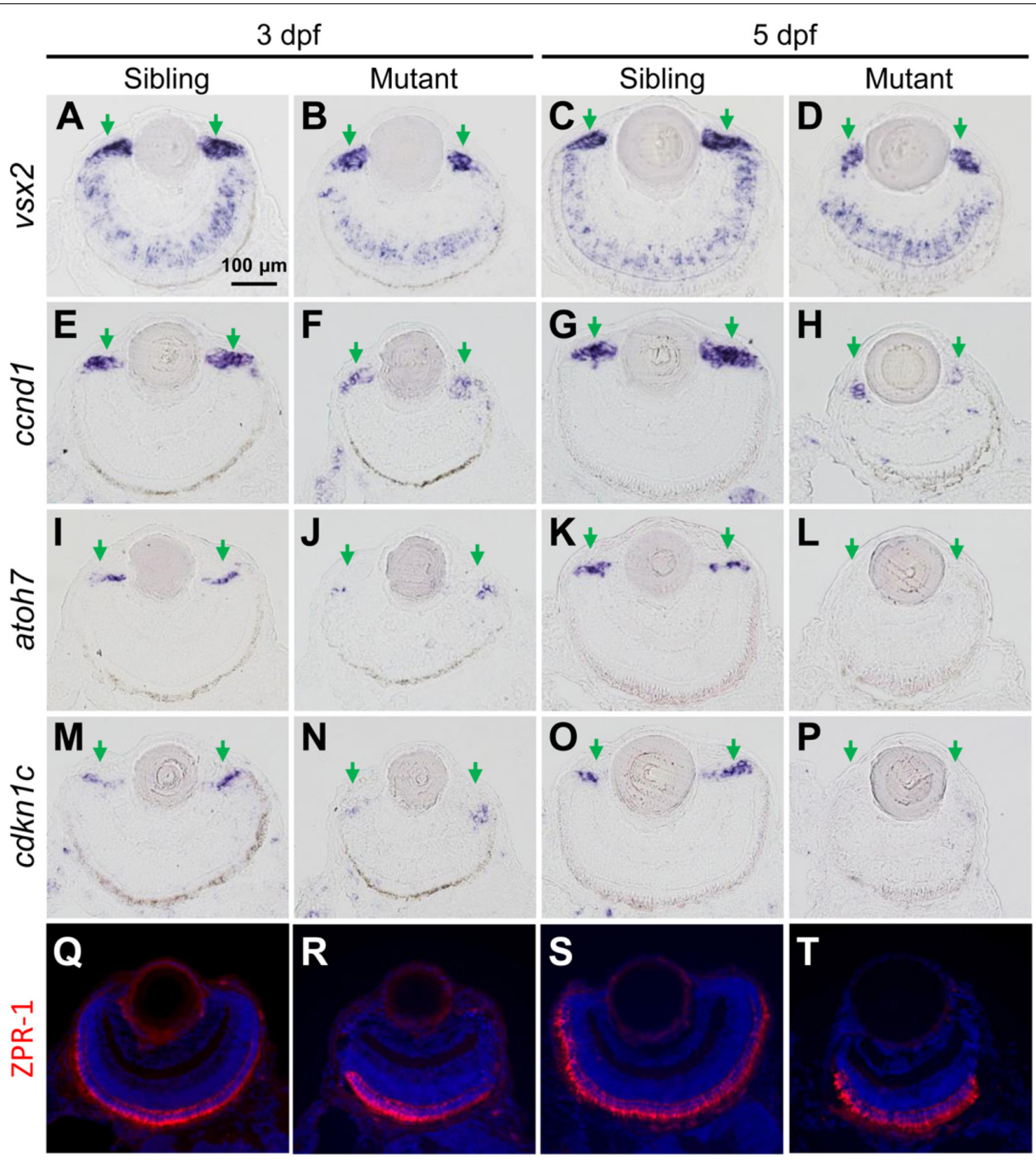

FIGURE 4 | The tubgcp3 mutant embryos exhibit decreased expression of proliferation and differentiation markers in CMZ cells. (A-D) ISH analyses show that the expression of vsX2 is normal in the central retina but significantly reduced in the CMZ in tubgcp3 mutant retinae at 3 dpf and 5 dpf. (E-H) ISH analyses exhibit that ccnd1 is highly expressed in wild-type sibling CMZ (M,O) but clearly reduced in tubgcp3 mutant CMZ (N,P) at 3 dpf and 5 dpf. (I-P) atoh7 and cdkn1c (associated with retinal cell differentiation) are expressed in the central $\mathrm{CMZ}$ of wild-type sibling retina but significantly reduced in tubgcp3 mutant $\mathrm{CMZ}$ at $3 \mathrm{dpf}$ and almost disappeared at $5 \mathrm{dpf}$. (Q-T) Immunostaining analyses displaying normal ZPR-1 staining (green/red double cone photoreceptors marker) in the central area of the tubgcp3 mutant retina. Arrows indicate the CMZ of the retina. Scale bars: $100 \mu \mathrm{m}$ (A-T).

using anti-centrin (a marker for centriole) and anti-PH3. In wild-type siblings, the mitotic cells had a pair of centrioles (two centrioles per centrosome) at each spindle pole (Figure 50). In the tubgcp 3 mutants, the M-phase arrested cells had four centriole dots like the wild-type siblings (Figures 5P,Q). However, the distribution of centrioles was abnormal. They were located at the center of the cell (Figure 5P) or randomly scattered in the cell (Figure 5Q). These data suggest that the centrioles duplicate normally but fail to separate correctly in mitotic arrested RPCs in the tubgcp3 mutants.

Previous work has reported that Tubgcp3 interacts with $\gamma$-tubulin to form $\gamma$-TuRC complexes, which are located at the centrosome during mitosis (Pereira et al., 1998; Kollman et al., 2010; Figure 5U). We next detected whether the distribution of $\gamma$-tubulin was affected after Tubgcp3 depletion. Our immunostaining results showed that $\gamma$-tubulin located at each spindle pole during mitosis in wild-type siblings (Figure 5R). In contrast, the distribution of $\gamma$-tubulin was abnormal in the mitotic arrested RPCs in tubgcp3 mutants. Most of the arrested cells that displayed $\gamma$-tubulin focus in the center of the cells (Figure 5S). Others showed scattered $\gamma$-tubulin staining patterns (Figure 5T). In order to test whether the interaction between Tubgcp 3 and $\gamma$-tubulin is conserved in zebrafish, we performed co-Immunoprecipitation (co-IP) assays using HEK293 cells by co-transfecting Myc-tagged zebrafish Tubgcp3 and GFP-tagged zebrafish $\gamma$-tubulin. Co-IP assays showed that Tubgcp3 binds to $\gamma$-tubulin through its $\mathrm{C}$-terminal domain, which is consistent with previous studies (Figure 5V). 


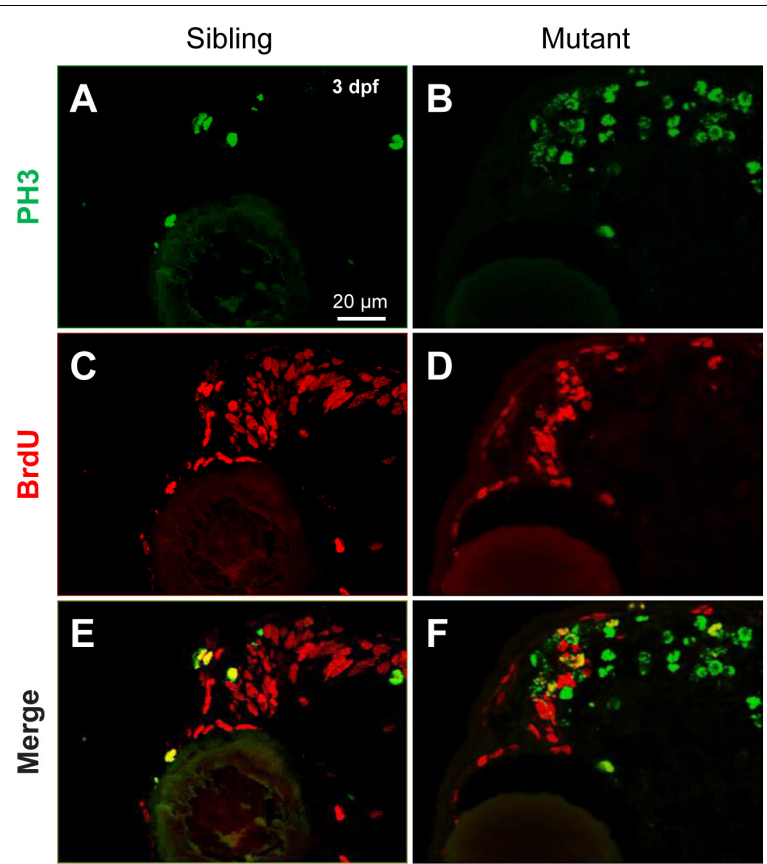

G

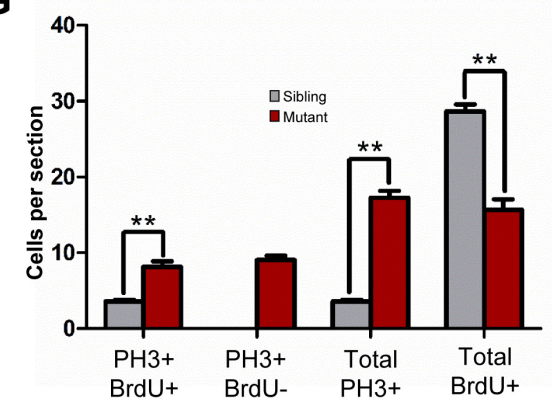

Sibling
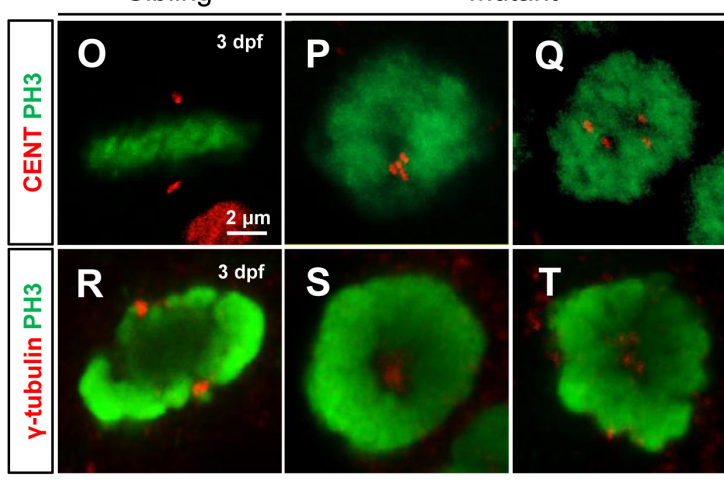

U

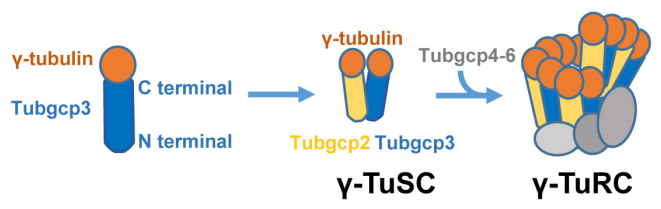

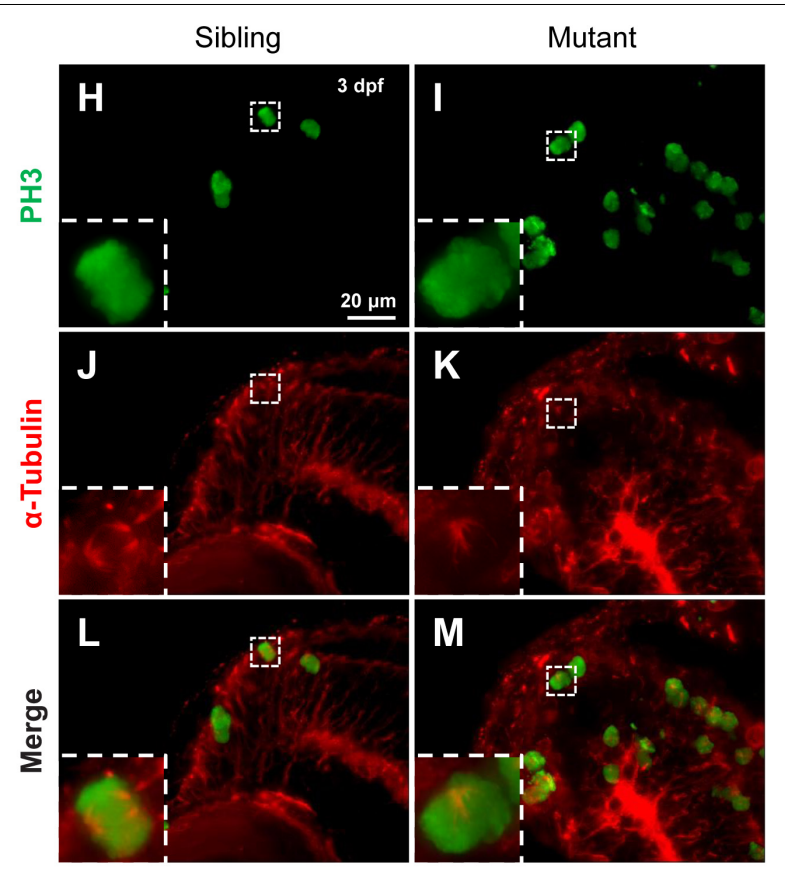

N

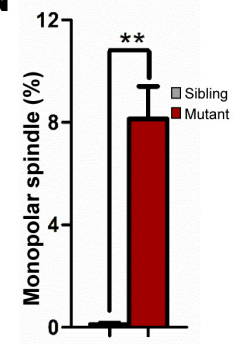

V

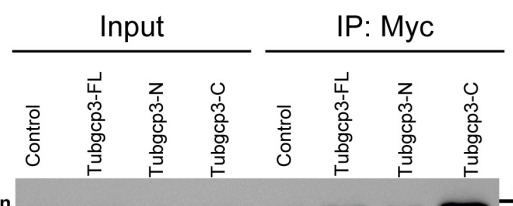

IB: GFP-y-tubulin _- _ _ - $-700 \mathrm{kDa}$

IB. Myc-Tubgcp3 $-100 \mathrm{kDa}$

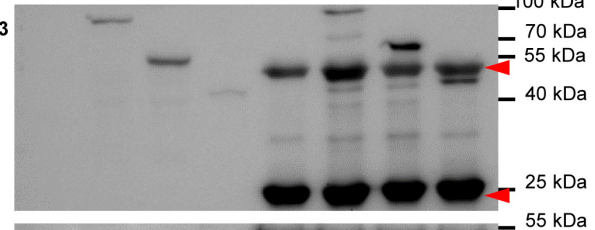

IB: $\beta$-Actin

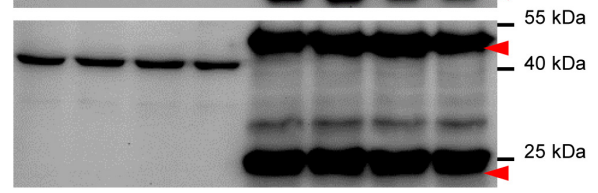

FIGURE 5 | The tubgcp3 mutant CMZ cells arrest in M-phase showing monopolar spindles and abnormal distributed centrioles and $\gamma$-tubulin. (A-F) Immunostaining analysis of cell proliferation in zebrafish retina at 3 dpf using DNA replication marker (BrdU, red) and mitotic marker (PH3, green). Embryos are incubated with BrdU for $6 \mathrm{~h}$ before being collected at $72 \mathrm{hpf}$ for the analysis. Almost all cells in wild-type sibling $\mathrm{CMZ}$ are BrdU+ with several PH3+ cells among them (A,C,E). In the 


\begin{abstract}
FIGURE 5 | Continued
tubgcp3 mutant retina, $\mathrm{PH} 3+$ cells are significantly increased (B,F), but BrdU+ cells are markedly decreased (D,F). Note that PH3+ BrdU- cells are detected in the tubgcp3 mutant retina (F) but absent in the wild-type sibling (E). (G) Bar chart analyses depicting quantification of BrdU- and PH3-labeled cells in wild-type sibling and tubgcp3 mutant retinae. Data are mean + SEM from 50 retinal sections for each group. Student's $t$-test: **P < 0.01 . (H-M) Immunostaining of 3 dpf retinal cryosections with anti- $\alpha$-tubulin (red) and anti-PH3 (green) displaying bipolar spindles formed in mitotic cells in wild-type siblings (H,J,L). In the tubgcp3 mutant retina, many mitotic RPCs exhibit monopolar spindles (I,K, $\mathbf{M})$. Insets indicate high-magnification images of mitotic RPCs in rectangles in (H-M). (N) Bar charts depicting quantification of mitotic cells with monopolar spindles in wild-type sibling ( 0.12 per section, $n=43$ sections) and the tubgcp3 mutant retinae ( 8.14 per section, $n=36$ sections). (O-Q) Immunostaining analyses displaying a pair of centrioles at each pole of the bipolar spindle in mitotic cells in wild-type sibling CMZ (0). In the tubgcp3 mutant retinae, centrioles are distributed at the center of the M-phase arrested cells (57.6\%, $n=59 \mathrm{M}$-phase arrested cells) (P) or randomly scatter in these cells (42.4\%, $n=59$ M-phase arrested cells) (Q). (R-T) Immunostaining analyses exhibiting $\gamma$-tubulin at the spindle poles in mitotic cells in wild-type sibling (R). In tubgcp3 mutant retinae, $\gamma$-tubulin localizes at the center of the M-phase arrested cells, showing a single focus (62.5\%, $n=80 \mathrm{M}$-phase arrested cells) (S) or scattered foci (37.5\%, $n=80 \mathrm{M}$-phase arrested cells) (T). (U) Schematic representation of the structure of $\gamma$-TuSC and $\gamma$-TuRC. (V) Co-immunoprecipitation (IP) assays showing Tubgcp3 interacts with $\gamma$-tubulin through its $\mathrm{C}$ terminal domain. HEK293T cells were transfected with plasmids to express GFP-tagged zebrafish $\gamma$-tubulin and Myc-tagged zebrafish Tubgcp3 fragments, including full length (1-906 aa) Tubgcp3, N terminal (1-551 aa) Tubgcp3 and C terminal (552-906 aa) Tubgcp3. Then the cell samples were performed by immunoprecipitation with anti-Myc antibody and analyzed by immunoblotting (IB) with anti-Myc and anti-GFP antibodies. $\beta$-Actin was used as the loading control. Arrowheads indicate the lgG heavy chain ( $\sim 50 \mathrm{kDa})$ and lgG light chain ( $25 \mathrm{kDa})$. Scale bars: $20 \mu \mathrm{m}(\mathbf{A}-\mathbf{F})$; $20 \mu \mathrm{m}$ (H-M); $2 \mu \mathrm{m}$ (O-T).
\end{abstract}

Taken together, our data suggest that depletion of Tubgcp3 does not affect centriole duplication, but results in abnormal distribution of centrioles and $\gamma$-tubulin.

\section{Tupgcp3-Deficient Cells Undergo Apoptosis and Senescence}

Given our HE staining results showed disappearance of cells in the tubgcp3 mutant CMZ from 3 to $5 \mathrm{dpf}$ (Figures $\mathbf{3 A - H}$ ), we next investigated whether the mitotic arrest RPCs underwent apoptosis using TUNEL assay. There were almost no TUNEL+ cells in wild-type siblings (Figures 6C,E,G,I). In contrast, TUNEL+ cells were significantly increased in tubgcp3 mutant $\mathrm{CMZ}$ and the adjacent regions (Figures $\mathbf{6 D}, \mathbf{F}, \mathbf{H}, \mathbf{I}$ ) at $3 \mathrm{dpf}$. However, the TUNEL+ cells and PH3 + cells did not co-localize in the mutants (Figures $\mathbf{6 F}, \mathbf{H}$ ). This may be due to the fact that PH3 expression was absent in late-stage apoptotic cells (Huang et al., 2006). Next, we performed immunostaining using $\gamma$-H2AX, an early stage apoptotic marker (Rogakou et al., 2000), together with PH3. There was a significant increase in the number of $\gamma$-H2AX + cells in the tubgcp3 mutant retina at $3 \mathrm{dpf}$ (Figures $\mathbf{6 M}, \mathbf{O}, \mathbf{Q}, \mathbf{R}$ ), compared to wild-type siblings (Figures 6L,N,P,R). Moreover, some of the $\gamma-\mathrm{H} 2 \mathrm{AX}+$ cells overlapped with $\mathrm{PH} 3+$ cells (Figures 6O,Q). These results suggest that the M-phase arrested RPCs undergo apoptosis in the tubgcp3 mutants.

In addition, RSCs [marked by col15a1b (Pujic et al., 2006; Cerveny et al., 2010; Gonzalez-Nunez et al., 2010; Valdivia et al., 2016)] still could be detected at the most peripheral CMZ (close to the lens) in the tubgcp3 mutant retina at $5 \mathrm{dpf}$ (Supplementary Figure S4A). Apoptosis signal was also absent in this region at $3 \mathrm{dpf}$ (Figures 6H,Q) and $5 \mathrm{dpf}$ (Supplementary Figure S6). We next want to detect cell proliferation at the peripheral CMZ. Embryos were incubated with BrdU for $24 \mathrm{~h}$ before being fixed at $5 \mathrm{dpf}$ for the assay. In this region, BrdU+ cells were significantly decreased in the tubgcp3 mutants compared to wild-type siblings (Figures 6S-T",Y). As the proliferation of RSCs occurs at the peripheral $\mathrm{CMZ}$, we speculated that RSC proliferation might be affected in the tubgcp 3 mutant CMZ. Previous studies have reported that centrosome dysfunction caused by centrosomal protein depletion could induce cellular senescence (Manning and
Kumar, 2010; Schmidt et al., 2010; Hossain and Tsang, 2013). Consistent with these reports, we found that the expression of senescence-associated $\beta$-galactosidase (SA- $\beta$-gal), a widely used senescent marker (Dimri et al., 1995), was significantly increased at the peripheral CMZ in the tubgcp3 mutant CMZ compared to the wild-type siblings (Figures 6U-X). These results indicate that depletion of Tubgcp3 caused cellular senescence at the peripheral CMZ that contains RSCs.

\section{DISCUSSION}

In this study, we generated the zebrafish tubgcp3 mutant and investigated the function of tubgcp3 in eye development. Our data reveal that Tubgcp3 plays important roles in cell cycle progression in the CMZ of the retina. Depletion of Tubgcp3 in RPCs results in monopolar spindle formation and impairs the distribution of centrioles and $\gamma$-tubulin, causing $M$-phase arrest and further apoptosis. In addition, depletion of Tubgcp3 results in cell proliferation defects and senescence at the peripheral CMZ. These defects led to the small eye phenotype in the tubgcp3 mutants. To our knowledge, this is the first in vivo model for the functional study of Tubgcp3. Our findings also provide some clues for studying the roles of its associated complexes, $\gamma$-TuSC and $\gamma$-TuRC, in vertebrate development.

$\gamma$-TuSC, composed of Tubgcp3, Tubgcp2, and $\gamma$-tubulin, is an essential complex for microtubule nucleation and spindle assembly during mitosis. Each component of $\gamma$-TuSC is likely essential for all cell proliferation in the developing zebrafish. Recently, Pouchucq et al. (2018) reported that morpholino (MO)-mediated tubg1/ $\gamma$-tubulin knockdown caused zebrafish embryo development arrest at the mid-gastrula stage. The early developmental defects are most probably attributable to the fact that MO was designed against the translation start site of tubg1 mRNA, which blocks the translation of both the maternal and zygotic tubg1 mRNA (Pouchucq et al., 2018). Although cell cycle defects and increased apoptosis were observed after depletion of $\gamma$-tubulin (Pouchucq et al., 2018), study on the function of $\gamma$-tubulin in later stages of development has been limited. In contrast, the tubgcp3 mutants can survive longer than 

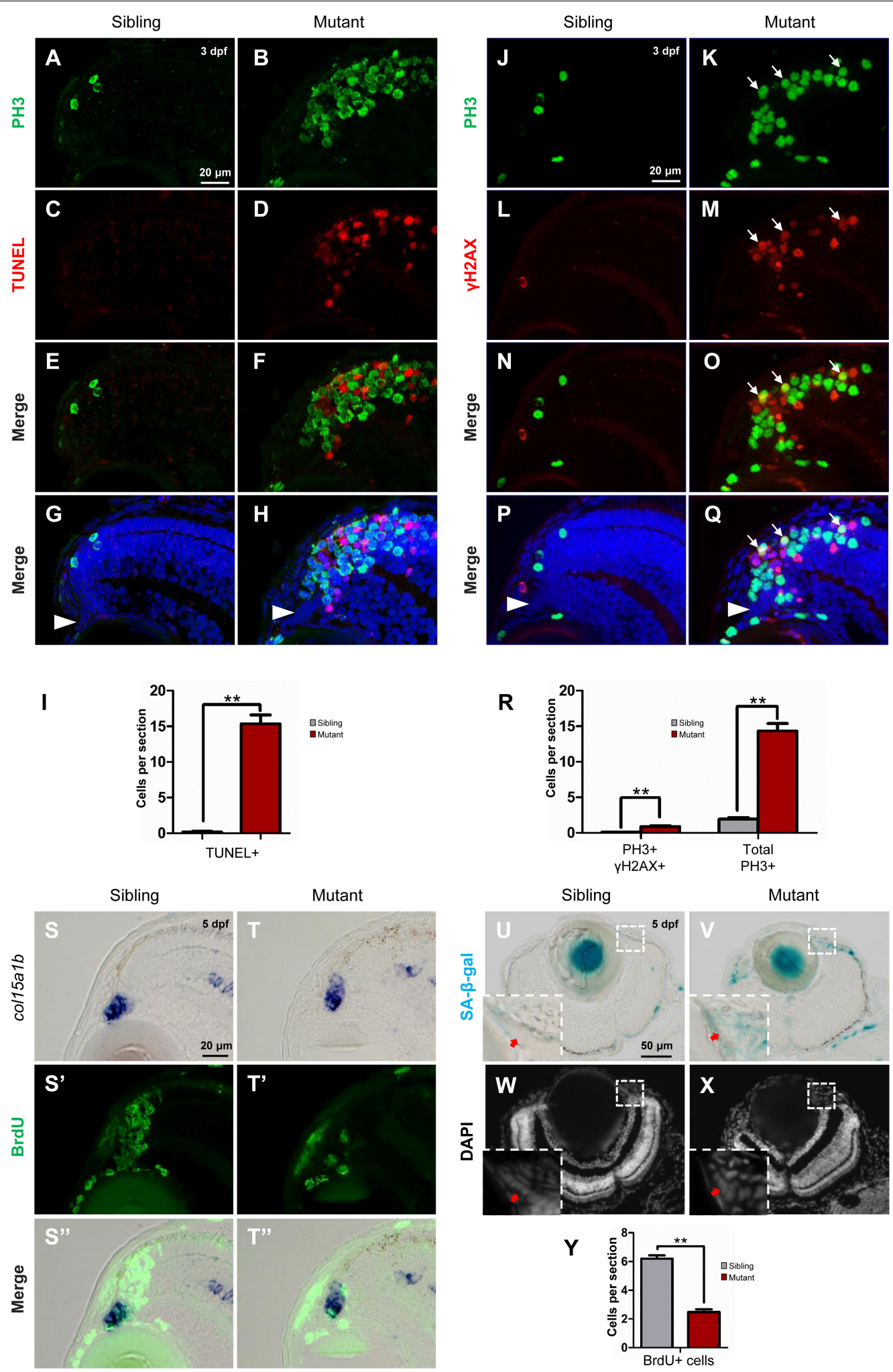

FIGURE 6 | Tupgcp3 deficient cells undergo apoptosis and senescence. (A-H) Immunostaining analyses displaying significantly increased TUNEL+ cells in the tubgcp3 mutant retina $\mathbf{( D , F , H )}$ at 3 dpf compared to wild-type sibling $\mathbf{( C , E , G ) . ~ N o t e ~ t h e ~ i n c r e a s e d ~} \mathrm{PH} 3+$ cells in the tubgcp3 mutant retina do not co-localize with the TUNEL+ cells (F,H). (I) Bar chart analyses depicting quantification of TUNEL+ cells in wild-type sibling and the tubgcp3 mutant retinae. (J-Q) Immunostaining analyses exhibiting markedly increased $\gamma-\mathrm{H} 2 \mathrm{AX}+$ cells in the tubgcp3 mutant retina $\mathbf{( M , O , Q )}$ at $3 \mathrm{dpf}$ compared to wild-type sibling (L,N,P). Note that some of the 


\section{FIGURE 6 | Continued}

increased $\mathrm{PH} 3+$ cells overlap with the $\gamma-\mathrm{H} 2 \mathrm{AX}+$ cells in the tubgcp3 mutant $(\mathbf{O}, \mathbf{Q})$. Arrows mark the $\mathrm{PH} 3$ and $\gamma-\mathrm{H} 2 \mathrm{AX}$ double positive cells in the tubgcp3 mutant retina. Arrowheads indicate the location of RSCs in the CMZ of the retina. (R) Bar chart analyses depicting quantification of $\gamma$-H2AX+ and PH3+ cells in wild-type sibling and the tubgcp3 mutant retinae. Data are mean + SEM from 30 sections for each group. Student's $t$-test: ** $P<0.01$. (S-T") ISH and immunostaining analysis of cell proliferation at the extreme periphery of CMZ at $5 \mathrm{dpf}$. Zebrafish embryos were incubated in BrdU for $24 \mathrm{~h}$ before collected at $5 \mathrm{dpf}$ for the double staining assay. In the wild-type sibling, there are many BrdU+ cells in co/15a1b-labeled region (S, $\mathbf{S}, \mathbf{S}$ '). In contrast, BrdU+ cells are significantly decreased in this region in the tubgcp3 mutant CMZ (T,T', T'). (U-X) Senescence-associated $\beta$-galactosidase (SA- $\beta$-gal) staining exhibiting increased $\beta$-galactosidase activity at the CMZ in the tubgcp3 mutant (V) compared to the wild-type sibling (U). Nuclei are stained with DAPI (W,X). Insets indicate high-magnification images of the peripheral edge of $\mathrm{CMZ}$ in rectangles in (U-X). (Y) Bar chart analyses depicting quantification of BrdU+ cells in the col15a1b-labeled region in wild-type sibling and the tubgcp3 mutant CMZ. Data are mean + SEM from 36 sections for each group. Student's t-test: ${ }^{* *} P<0.01$. Scale bars: $20 \mu \mathrm{m}$ (A-H); $20 \mu \mathrm{m}$ (J-Q); $20 \mu \mathrm{m}$ (S-T”); $50 \mu \mathrm{m}(\mathbf{U}-\mathbf{X})$.

10 days due to the maternal deposition of tubgcp3 gene products. The maternal effect provides a possibility to study its function at relatively late developmental stages. In the tubgcp3 mutant, the differentiated cells seem to be unaffected (Figures 3I-L). However, the CMZ exhibits obvious defects from 3 to $5 \mathrm{dpf}$ (Figures 3E-H). This is associated with the relatively higher expression of tubgcp 3 in $\mathrm{CMZ}$ compared to other retinal regions (Figures 1L,M). In CMZ, new neurons are produced for the continuous growth of zebrafish eyes after the embryogenesis of retina completed at $60 \mathrm{hpf}$. The tissue-specific defects in CMZ are in accordance with the rapid depletion of the maternal store in the regions of high proliferation. This also correlates with the function of $\gamma$-TuSC in cell proliferation.

Despite the fact that many studies have been carried out among different organisms to understand the function of Tubgcp 3 in $\gamma$-TuRC assembly and centrosome function (Geissler et al., 1996; Barbosa et al., 2000; Mikule et al., 2007; Xiong and Oakley, 2009; Farache et al., 2016; Cota et al., 2017), its roles in vertebrate development remain unclear. In vitro studies using human cell lines show that GCP3/Tubgcp3 is required for cell cycle progression (Mikule et al., 2007; Draberova et al., 2015; Cota et al., 2017). However, the cell cycle defects caused by GCP3 depletion are conflicting. Draberova et al. (2015) reported that the wild-type p53 U87MG cells and mutant p53 T98 cells arrested in M-phase after depletion of GCP3 using siRNA. GCP3-depleted HeLa cells also arrested in the M-phase (Cota et al., 2017). In the tubgcp 3 mutants, we found that RPCs arrested in M-phase in the CMZ of the retina (Figures 5B,D,F,G), which is consistent with previous studies. Moreover, the mitotic arrest RPCs underwent apoptosis (Figures 6A-R). However, Mikule et al. (2007) found that some GCP3-depleted cell lines, such as RPE-1, BJ-1, HME1, and HCT-116, did not arrest in M-phase, but arrested in G1 phase in a p53-dependent manner. In our study, we found that cell proliferation was affected at the peripheral CMZ in the tubgcp 3 mutant retina. These cells might be RSCs, which failed to progress into S phase (Figures $\mathbf{6 S - T}$, Y). They did not arrest in $\mathrm{M}$-phase as $\mathrm{PH} 3+$ cells did not accumulate in the mutant peripheral CMZ at $5 \mathrm{dpf}$ (Supplementary Figure S7). It is not feasible for us to isolate RSCs for fluorescence-activated cell sorter analysis (FACS) due to the absence of available transgenic zebrafish lines expressing RSC-specific reporters in our lab. Whether depletion of Tubgcp3 in RSCs causes G1 arrest still needs further study. To study whether p53 is involved in these processes will be an important extension to the functional study of Tubgcp3. It has been reported that centrosome dysfunction could induce cellular senescence (Manning and Kumar, 2010; Schmidt et al., 2010; Hossain and Tsang, 2013). Consistent with these reports, we observed that some cells underwent senescence at the extreme periphery of CMZ in tubgcp3 mutants. Since CMZ cells are involved in the growth of zebrafish eye, these defects together caused the small eye phenotype in the tubgcp3 mutants.

Autosomal recessive primary microcephaly (MCPH) is a neurodevelopmental disorder characterized by markedly reduced brain size. The patients exhibited significantly reduced number of neural progenitor cells caused by cell proliferation defects, increased cell death and the disruption of the balance between symmetric and asymmetric division (Barbelanne and Tsang, 2014; Buchwalter et al., 2016; O’Neill et al., 2018). Currently, at least nine centrosome-related genes have been genetically linked to microcephaly disorders (Megraw et al., 2011; Novorol et al., 2013; Chavali et al., 2014; Morris-Rosendahl and Kaindl, 2015; Buchwalter et al., 2016). Mutations in tubgcp4 and tubgcp6 have been reported to be associated with MCPH (Buchwalter et al., 2016). They encode TUBGCP4 and TUBGCP6, respectively, two components of $\gamma$-TuRC (Zheng et al., 1995). In addition, the MCPH models have been established using zebrafish retinal neuroepithelium by depletion of centrosomal proteins, including STIL, ASPM, WDR62, and ODF2 (Novorol et al., 2013). STIL localizes to the procentriolar cartwheel region and plays important roles in centriole duplication (Arquint and Nigg, 2014). ASPM localizes at the spindle poles and is involved in the organization of spindle poles (Tungadi et al., 2017). WDR62, which also localizes to the spindle poles, is required for mitotic entry of neural stem cells (Ramdas Nair et al., 2016). ODF2, a mother centriole subdistal appendage protein, is indispensable for the assembly of the mother centriole (Ishikawa et al., 2005). In these models, zebrafish embryos display the $\mathrm{MCPH}$ phenotype with significantly reduced head and eye size (Novorol et al., 2013). After knockout of tubgcp3 gene, the zebrafish embryos exhibit the similar MCPH phenotype (Figures 2E-G). M-phase cell cycle arrest (Figures 5A-G) and increased apoptosis are also observed (Figures 6A-R) in tubgcp3 mutant retinae, which is consistent with these $\mathrm{MCPH}$ models (Novorol et al., 2013). Moreover, cell proliferation defect and senescence were also observed at the peripheral CMZ (stem cell niche) (Figures 6S-V,Y). Senescent cells were also observed in the brain of the tubgcp3 mutants (Supplementary Figure S8). Given the MCPH phenotype observed in tubgcp3 mutants (Figures 2E-G) and the requirement of Tubgcp3 in $\gamma$-TuRC 
assembly and centrosome function (Geissler et al., 1996; Barbosa et al., 2000; Mikule et al., 2007; Xiong and Oakley, 2009; Farache et al., 2016; Cota et al., 2017), we speculate that premature senescence may provide a possible new view to explain the mechanism of MCPH. Further studies to characterize the function of other centrosomal proteins will be necessary to fully understand the relationship between centrosome and microcephaly disorders.

\section{ETHICS STATEMENT}

This study was carried out according to the Guide for the Care and Use of Laboratory Animals from the National Institutes of Health. The experimental protocol was approved by the Animal Care and Use Committee of Fudan University.

\section{AUTHOR CONTRIBUTIONS}

TZ conceived and directed the project. GL carried out the experiments and discovered the roles of Tubgcp3 in cell cycle progression during zebrafish retinal development. TZ, DJ, and GL prepared the figures and wrote the manuscript.

\section{REFERENCES}

Arquint, C., and Nigg, E. A. (2014). STIL microcephaly mutations interfere with APC/C-mediated degradation and cause centriole amplification. Curr. Biol. 24, 351-360. doi: 10.1016/j.cub.2013.12.016

Barbelanne, M., and Tsang, W. Y. (2014). Molecular and cellular basis of autosomal recessive primary microcephaly. Biomed. Res. Int. 2014:547986. doi: 10.1155/ 2014/547986

Barbosa, V., Yamamoto, R. R., Henderson, D. S., and Glover, D. M. (2000). Mutation of a drosophila gamma tubulin ring complex subunit encoded by discs degenerate- 4 differentially disrupts centrosomal protein localization. Genes Dev. 14, 3126-3139. doi: 10.1101/gad.182800

Bornens, M. (2002). Centrosome composition and microtubule anchoring mechanisms. Curr. Opin. Cell Biol. 14, 25-34. doi: 10.1016/S0955-0674(01) 00290-3

Buchwalter, R. A., Chen, J. V., Zheng, Y., and Megraw, T. L. (2016). Centrosome in cell division, development and disease. eLS 30, 1-2.

Cerveny, K. L., Cavodeassi, F., Turner, K. J., de Jong-Curtain, T. A., Heath, J. K., and Wilson, S. W. (2010). The zebrafish flotte lotte mutant reveals that the local retinal environment promotes the differentiation of proliferating precursors emerging from their stem cell niche. Development 137, 2107-2115. doi: 10.1242/ dev.047753

Chavali, P. L., Putz, M., and Gergely, F. (2014). Small organelle, big responsibility: the role of centrosomes in development and disease. Philos. Trans. R. Soc. Lond. B Biol. Sci. 369:20130468. doi: 10.1098/rstb.2013.0468

Cota, R. R., Teixido-Travesa, N., Ezquerra, A., Eibes, S., Lacasa, C., Roig, J., et al. (2017). MZT1 regulates microtubule nucleation by linking gammaTuRC assembly to adapter-mediated targeting and activation. J. Cell Sci. 130, 406-419. doi: $10.1242 /$ jcs.195321

Dimri, G. P., Lee, X. H., Basile, G., Acosta, M., Scott, C., Roskelley, C., et al. (1995). A biomarker that identifies senescent human-cells in culture and in aging skin In-Vivo. Proc. Natl. Acad. Sci. U.S.A. 92, 9363-9367. doi: 10.1073/pnas.92.20. 9363

Draberova, E., D’Agostino, L., Caracciolo, V., Sladkova, V., Sulimenko, T., Sulimenko, V., et al. (2015). Overexpression and nucleolar localization of gamma-tubulin small complex proteins GCP2 and GCP3 in

\section{FUNDING}

This work was supported by grants from the National Science Foundation of China (31530044, 31671514, and 31471357).

\section{ACKNOWLEDGMENTS}

We acknowledge Haitao Zhou for excellent fish care. The transgenic line $\mathrm{Tg}(\mathrm{HuC:GFP})$ was kindly provided by $\mathrm{Su}$ Guo (University of California). We are grateful to laboratory members Ruilin Zhang (Fudan University), Su Guo (University of California), Jie He (Chinese Academy of Sciences), and Ying Cao (Tongji University) for technical advice. We thank members of our laboratory for helpful discussions. We also thank the China Zebrafish Resource Center (CZRC) and the Zebrafish All Genes Knockout Consortium (ZAKOC) for important cooperation and assistance.

\section{SUPPLEMENTARY MATERIAL}

The Supplementary Material for this article can be found online at: https://www.frontiersin.org/articles/10.3389/fnmol. 2019.00126/full\#supplementary-material

glioblastoma. J. Neuropathol. Exp. Neurol. 74, 723-742. doi: 10.1097/NEN. 0000000000000212

Farache, D., Jauneau, A., Chemin, C., Chartrain, M., Remy, M. H., Merdes, A., et al. (2016). Functional analysis of gamma-tubulin complex proteins indicates specific lateral association via their N-terminal domains. J. Biol. Chem. 291, 23112-23125. doi: 10.1074/jbc.M116.744862

Geissler, S., Pereira, G., Spang, A., Knop, M., Soues, S., Kilmartin, J., et al. (1996). The spindle pole body component Spc98p interacts with the gamma-tubulinlike Tub4p of Saccharomyces cerevisiae at the sites of microtubule attachment. EMBO J. 15, 3899-3911. doi: 10.1002/j.1460-2075.1996.tb00764.x

Gonzalez-Nunez, V., Nocco, V., and Budd, A. (2010). Characterization of drCol 15alb: a novel component of the stem cell niche in the zebrafish retina. Stem Cells 28, 1399-1411. doi: 10.1002/stem.461

Hossain, D., and Tsang, W. Y. (2013). Centrosome dysfunction and senescence: coincidence or causality? J. Aging Sci. 1:3. doi: 10.4172/2329-8847.1000113

Huang, X., Kurose, A., Tanaka, T., Traganos, F., Dai, W., and Darzynkiewicz, Z. (2006). Sequential phosphorylation of Ser-10 on histone H3 and ser-139 on histone H2AX and ATM activation during premature chromosome condensation: relationship to cell-cycle phase and apoptosis. Cytometry A 69, 222-229. doi: 10.1002/cyto.a.20257

Ishikawa, H., Kubo, A., Tsukita, S., and Tsukita, S. (2005). Odf2-deficient mother centrioles lack distal/subdistal appendages and the ability to generate primary cilia. Nat. Cell Biol. 7, 517-524. doi: 10.1038/ncb1251

Kellogg, D. R., Moritz, M., and Alberts, B. M. (1994). The centrosome and cellular organization. Annu. Rev. Biochem. 63, 639-674. doi: 10.1146/annurev.bi.63. 070194.003231

Knop, M., Pereira, G., Geissler, S., Grein, K., and Schiebel, E. (1997). The spindle pole body component Spc97p interacts with the gamma-tubulin of Saccharomyces cerevisiae and functions in microtubule organization and spindle pole body duplication. EMBO J. 16, 1550-1564. doi: 10.1093/emboj/16. 7.1550

Kollman, J. M., Polka, J. K., Zelter, A., Davis, T. N., and Agard, D. A. (2010). Microtubule nucleating gamma-TuSC assembles structures with 13-fold microtubule-like symmetry. Nature 466, 879-882. doi: 10.1038/nature09207

Li, Z., Joseph, N. M., and Easter, S. S. Jr. (2000). The morphogenesis of the zebrafish eye, including a fate map of the optic vesicle. Dev. Dyn. 218, 175-188. 
Manning, J. A., and Kumar, S. (2010). A potential role for NEDD1 and the centrosome in senescence of mouse embryonic fibroblasts. Cell Death Dis. 1:e35. doi: $10.1038 /$ cddis. 2010.12

Marcus, R. C., Delaney, C. L., and Easter, S. S. Jr. (1999). Neurogenesis in the visual system of embryonic and adult zebrafish (Danio rerio). Vis. Neurosci. 16, 417-424. doi: 10.1017/s095252389916303x

Masai, I., Stemple, D. L., Okamoto, H., and Wilson, S. W. (2000). Midline signals regulate retinal neurogenesis in zebrafish. Neuron 27, 251-263. doi: 10.1016/ S0896-6273(00)00034-9

Megraw, T. L., Sharkey, J. T., and Nowakowski, R. S. (2011). Cdk5rap2 exposes the centrosomal root of microcephaly syndromes. Trends Cell Biol. 21, 470-480. doi: 10.1016/j.tcb.2011.04.007

Mikule, K., Delaval, B., Kaldis, P., Jurcyzk, A., Hergert, P., and Doxsey, S. (2007). Loss of centrosome integrity induces p38-p53-p21-dependent G1-S arrest. Nat. Cell Biol. 9, 160-170.

Morris-Rosendahl, D. J., and Kaindl, A. M. (2015). What next-generation sequencing (NGS) technology has enabled us to learn about primary autosomal recessive microcephaly (MCPH). Mol. Cell. Probes 29, 271-281. doi: 10.1016/j. mcp.2015.05.015

Novorol, C., Burkhardt, J., Wood, K. J., Iqbal, A., Roque, C., Coutts, N., et al. (2013). Microcephaly models in the developing zebrafish retinal neuroepithelium point to an underlying defect in metaphase progression. Open Biol. 3:130065. doi: 10.1098/rsob.130065

Oakley, B. R., Oakley, C. E., Yoon, Y. S., and Jung, M. K. (1990). Gamma-tubulin is a component of the spindle pole body that is essential for microtubule function in aspergillus-nidulans. Cell 61, 1289-1301. doi: 10.1016/0092-8674(90)90 693-9

Ohnuma, S., Philpott, A., Wang, K., Holt, C. E., and Harris, W. A. (1999). p27Xic1, a Cdk inhibitor, promotes the determination of glial cells in xenopus retina. Cell 99, 499-510. doi: 10.1016/S0092-8674(00)81538-X

O’Neill, R. S., Schoborg, T. A., and Rusan, N. M. (2018). Same but different: pleiotropy in centrosome-related microcephaly. Mol. Biol. Cell 29, 241-246. doi: 10.1091/mbc.E17-03-0192

Park, H. C., Kim, C. H., Bae, Y. K., Yeo, S. Y., Kim, S. H., Hong, S. K., et al. (2000). Analysis of upstream elements in the $\mathrm{HuC}$ promoter leads to the establishment of transgenic zebrafish with fluorescent neurons. Dev. Biol. 227, 279-293. doi: $10.1006 /$ dbio. 2000.9898

Pelegri, F. (2003). Maternal factors in zebrafish development. Dev. Dyn. 228, 535-554. doi: 10.1002/dvdy.10390

Pereira, G., Knop, M., and Schiebel, E. (1998). Spc98p directs the yeast gammatubulin complex into the nucleus and is subject to cell cycle-dependent phosphorylation on the nuclear side of the spindle pole body. Mol. Biol. Cell 9, 775-793. doi: 10.1091/mbc.9.4.775

Pouchucq, L., Undurraga, C. A., Fuentes, R., Cornejo, M., Allende, M. L., and Monasterio, O. (2018). $\gamma$-Tubulin small complex formation is essential for early zebrafish embryogenesis. Mechan. Dev. 154, 145-152. doi: 10.1016/j.mod.2018. 06.006

Pujic, Z., Omori, Y., Tsujikawa, M., Thisse, B., Thisse, C., and Malicki, J. (2006). Reverse genetic analysis of neurogenesis in the zebrafish retina. Dev. Biol. 293, 330-347. doi: 10.1016/j.ydbio.2005.12.056

Ramdas Nair, A., Singh, P., Salvador Garcia, D., Rodriguez-Crespo, D., Egger, B., and Cabernard, C. (2016). The microcephaly-associated protein Wdr62/CG7337 is required to maintain centrosome asymmetry in drosophila neuroblasts. Cell Rep. 14, 1100-1113. doi: 10.1016/j.celrep.2015.12.097

Rogakou, E. P., Nieves-Neira, W., Boon, C., Pommier, Y., and Bonner, W. M. (2000). Initiation of DNA fragmentation during apoptosis induces phosphorylation of H2AX histone at serine 139. J. Biol. Chem. 275, 9390-9395. doi: 10.1074/jbc.275.13.9390

Schmidt, S., Schneider, L., Essmann, F., Cirstea, I. C., Kuck, F., Kletke, A., et al. (2010). The centrosomal protein TACC3 controls paclitaxel sensitivity by modulating a premature senescence program. Oncogene 29, 6184-6192. doi: 10.1038 /onc. 2010.354

Shkumatava, A., and Neumann, C. J. (2005). Shh directs cell-cycle exit by activating p57Kip2 in the zebrafish retina. EMBO Rep. 6, 563-569. doi: 10.1038/sj.embor. 7400416

Thisse, C., and Thisse, B. (2008). High-resolution in situ hybridization to whole-mount zebrafish embryos. Nat. Protoc. 3, 59-69. doi: 10.1038/nprot.200 7.514

Tungadi, E. A., Ito, A., Kiyomitsu, T., and Goshima, G. (2017). Human microcephaly ASPM protein is a spindle pole-focusing factor that functions redundantly with CDK5RAP2. J. Cell. Sci. 130, 3676-3684. doi: 10.1242/jcs. 203703

Valdivia, L. E., Lamb, D. B., Horner, W., Wierzbicki, C., Tafessu, A., Williams, A. M., et al. (2016). Antagonism between Gdf6a and retinoic acid pathways controls timing of retinal neurogenesis and growth of the eye in zebrafish. Development 143, 1087-1098. doi: 10.1242/dev.130922

Vitorino, M., Jusuf, P. R., Maurus, D., Kimura, Y., Higashijima, S., and Harris, W. A. (2009). Vsx2 in the zebrafish retina: restricted lineages through derepression. Neural Dev. 4:14. doi: 10.1186/1749-8104-4-14

Wan, Y., Almeida, A. D., Rulands, S., Chalour, N., Muresan, L., Wu, Y., et al. (2016). The ciliary marginal zone of the zebrafish retina: clonal and timelapse analysis of a continuously growing tissue. Development 143, 1099-1107. doi: 10.1242/dev.133314

Wehman, A. M., Staub, W., and Baier, H. (2007). The anaphase-promoting complex is required in both dividing and quiescent cells during zebrafish development. Dev. Biol. 303, 144-156. doi: 10.1016/j.ydbio.2006. 10.043

Wehman, A. M., Staub, W., Meyers, J. R., Raymond, P. A., and Baier, H. (2005). Genetic dissection of the zebrafish retinal stem-cell compartment. Dev. Biol. 281, 53-65. doi: 10.1016/j.ydbio.2005.02.010

Westerfield, M. (2000). The Zebrafish Book. A Guide for the Laboratory Use of Zebrafish (Danio rerio). Eugene, OR: University of Oregon Press.

Xiong, Y., and Oakley, B. R. (2009). In vivo analysis of the functions of gammatubulin-complex proteins. J. Cell. Sci. 122(Pt 22), 4218-4227. doi: 10.1242/jcs. 059196

Yuba-Kubo, A., Kubo, A., Hata, M., and Tsukita, S. (2005). Gene knockout analysis of two gamma-tubulin isoforms in mice. Dev. Biol. 282, 361-373. doi: 10.1016/ j.ydbio.2005.03.031

Zheng, Y., Wong, M. L., Alberts, B., and Mitchison, T. (1995). Nucleation of microtubule assembly by a gamma-tubulin-containing ring complex. Nature 378, 578-583. doi: $10.1038 / 378578 \mathrm{a} 0$

Conflict of Interest Statement: The authors declare that the research was conducted in the absence of any commercial or financial relationships that could be construed as a potential conflict of interest.

Copyright (c) $2019 \mathrm{Li}$, Jin and Zhong. This is an open-access article distributed under the terms of the Creative Commons Attribution License (CC BY). The use, distribution or reproduction in other forums is permitted, provided the original author(s) and the copyright owner(s) are credited and that the original publication in this journal is cited, in accordance with accepted academic practice. No use, distribution or reproduction is permitted which does not comply with these terms. 